\title{
Co-Centric Cell-Splitting Technique Using Frequency Reuse
}

\author{
Ranjan Kumar Mondal ${ }^{1}$ and Debabrata Sarddar ${ }^{2}$ \\ ${ }^{1}$ Department of Computer Science \& Engineering, \\ University of Kalyani, Kalyani, India \\ ${ }^{2}$ Assistant Professor, Department of Computer Science \& Engineering, \\ University of Kalyani, Kalyani, India \\ ${ }^{1}$ ranjan@klyuniv.ac.in, ${ }^{2}$ dsarddar1@gmail.com
}

\begin{abstract}
When a MS or mobile node (MN) moves out from a base station (BS) to another base station then it needs to perform a handoff or handover. Here we will propose a method that minimizes the handoff failure probability by increasing the total number of channel with help cell splitting process. This is Mother and child cell concept.
\end{abstract}

Key Words: Access Point, Frequency Reuse, Cell Splitting, Channel, Mother Cell, Child Cell

\section{Introduction}

Handoff is very important issue in mobile communication. We know every mobile phone base station has limited channels that are spaced by $5 \mathrm{MHz}$ with a bandwidth of $22 \mathrm{MHz}$.

Many dynamic channel allocations have been proposed by different authors in different time and all those mechanisms of different authors will improve the performance of handoff in mobile communication. However for practical reason channel allocation is to be done in a static manner.

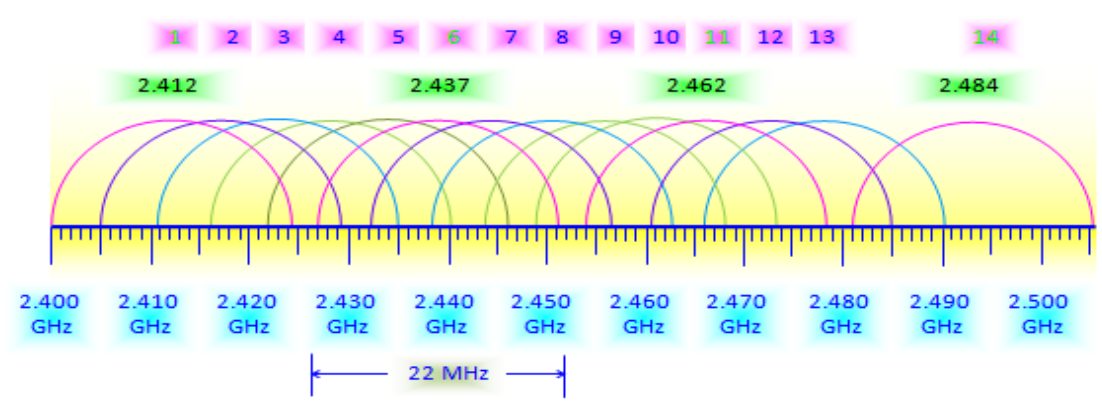

Figure 1. Channel Distribution

Channel allocation refers to the division of a given radio spectrum into a set of disjoint channels, which can be used simultaneously while minimizing interference in adjacent channels by good channel separation. There are three ways in which channel allocation is mainly done. They are:

a) Fixed Channel Allocation scheme

b) Dynamic Channel Allocation scheme

c) Hybrid Channel Allocation scheme

The rest of the paper would be done as follow:

We will describe the related works in the second section. We will also describe the details of proposed method in the third section. The simulation results of related cell 
splitting technique would be discussed in section four. In the next section we conclude the whole paper and finally a future work is mentioned regarding this paper in section six.

\section{Related Work}

The MS communicates to its BS by its channel (the channel being used by its current MS) which not only contains the neighbor of the AP on which it is being operated, but also the channels are used by the neighboring APs. However the MS must wait for required channel time as the MS does not know how many APs would respond to the probe request. So here we can use uni-cast AP instead of broadcast APs which selects the most potential AP to which the call may be handed off and scans only the channels associated with those APs. Selective channel probing with the help of unicast instead of broadcast brilliantly reduces the handoff delay by a massive percentage when compared with selective scanning or basic active scanning. Moreover, it was also stated that the MS has to wait for only the 'Round Trip Time' (RTT) for scanning each channel instead of the required channel time. When the MS responds to handoff, according to the prescanning mechanism of neighbor graph, it first looks for the potential AP.

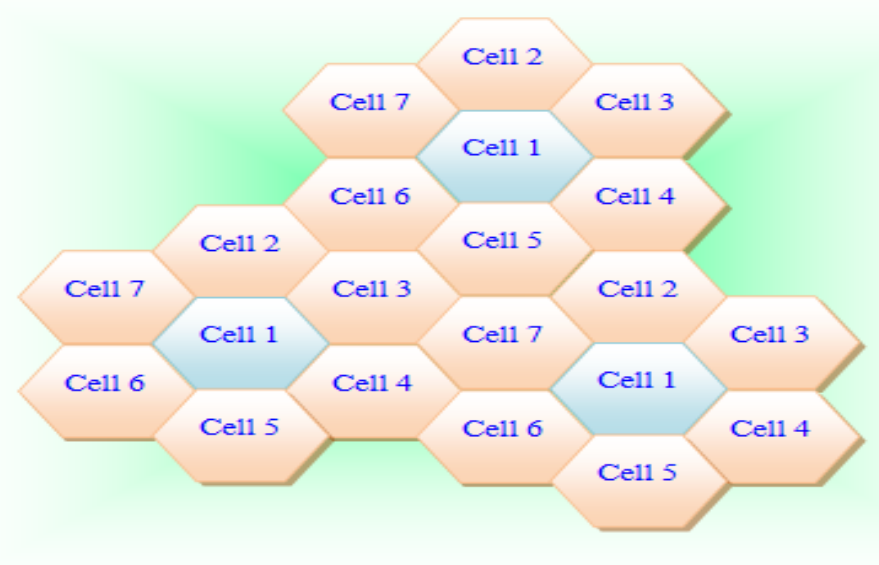

\section{Figure 2. Diagram Representing Cells That Can Use the Same Frequency Channel}

\subsection{Frequency Reuse}

Frequency reuse is a process of selecting and allocating channels for all of the BSs within a cellular network. The increased capacity in a cellular network, comparing to a network with a single transmitter, comes from the fact that the same radio frequency can be reused in a different area for a completely different transmission. If there is a single plain transmitter, only one transmission can be used on any given frequency. Unfortunately, there is inevitably some level of interference from the signal from the other cells which use the same frequency. This means that, in a standard FDMA system, there must be at least a one cell gap between cells which reuse the same frequency.

The frequency reuse factor is the rate where the same frequency can be used in the network. It is $1 / \mathrm{n}$ where $\mathrm{n}$ is the number of cells which cannot use a frequency for transmission.

\section{Proposed Work}

In our proposed work we can increase the channel capacity by using cell splitting. If we make a simplistic assumption that MS uniformly distributed in each cell, we can also say that the probability a channel being available in a new cell area depends on the 
number of channel per unit area. It can easily observe that the number of channels-area increase if the number of channel allocated per cell area increased. The radio resource and hence the number of assigned channel are limited and may not be change to be extent. However, the cell coverage area could be decreased for given number of channels per cells. This leads to smaller cell size and availability of free channel increase and then we allocate free channel using location manager based on call coverage area, duration etc.

\subsection{Cell Splitting:}

Cell splitting is the process of subdividing a large cell into some smaller cells, i.e., the total area of some smaller cells are equal to about the area of old larger cell in size, and each smaller cell will have with its own base station and a corresponding reduction in antenna with height and transmitter power. Cell splitting would increase the capacity of a cellular system since it increases the number of times that channels are reused.

By defining new cells which have a smaller radius than the original cells and by installing these smaller cells between the existing cells, capacity increases due to the additional number of channels per unit area. The consequence of the cell splitting is that the frequency assignment has to be done again, which affects the neighboring cells. It also increases the handoff rate because the cells are now smaller and a mobile is likely to cross cell boundaries more often compared with the case when the cells are big. Because of altered signaling conditions, this also affects the traffic in control channels.

An example of cell splitting is shown in Figure 3. Here it is assumed that the cell cluster is congested and as a result, the call blocking probability has risen above an acceptable level. Suppose every cell in the cluster will be reduced in such a way that the radius, $\mathrm{R}$ of every cell will be cut in half, $(\mathrm{R} / 2)$. In order to cover the entire service area with smaller cells, approximately two times as many cells would be required.

The increased number of cells would increase the number of clusters over the coverage region, which in turn would increase the number of channels, and thus capacity, in the coverage area. In the example shown in Figure 5, the smaller cells were added in such a way as to preserve the frequency reuse plan of the system. In this case, the radius of each new microcell is half that of the original cell.

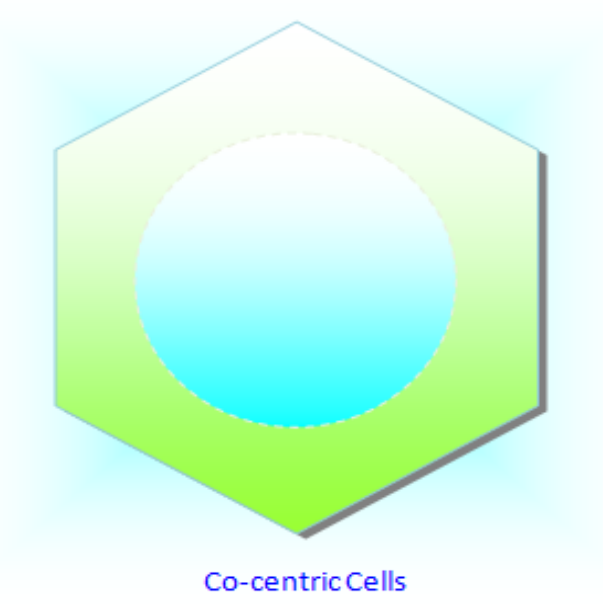

Figure 3. Structure of Microcell

From the above figure the radius of the outside cell is denoted by $R_{1}$ and the inner side cell is denoted by $R_{2}$. The ratio of radius of the two cells is $R_{2} / 2: R_{1}$ or $\left(R_{2}-R_{1}\right): R_{1}$ (i.e., $R_{2}=2 R_{1}$ ). Only non-overlapping outside area of outside cell is $\pi R_{2}^{2} / 4$. 


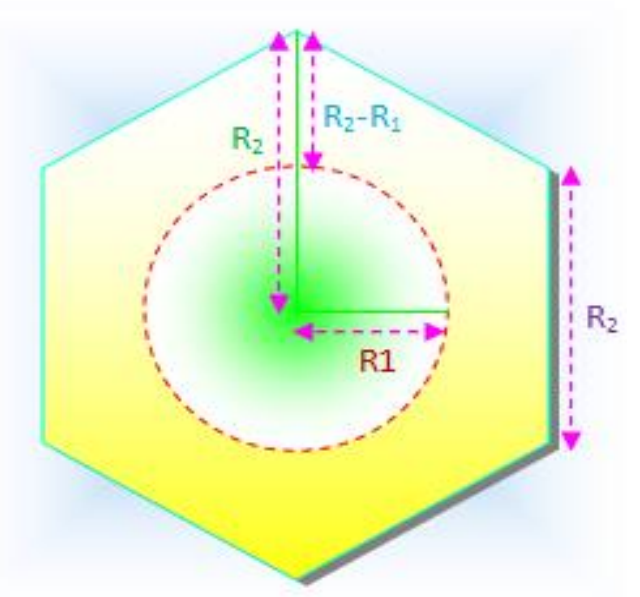

Figure 4. Area of Cells

Now the area ratio of radius $R_{2} / 2$ and $R_{1}$ cell is equal. This is because we want to subdivide all channel allocation to be equal for optimum value.

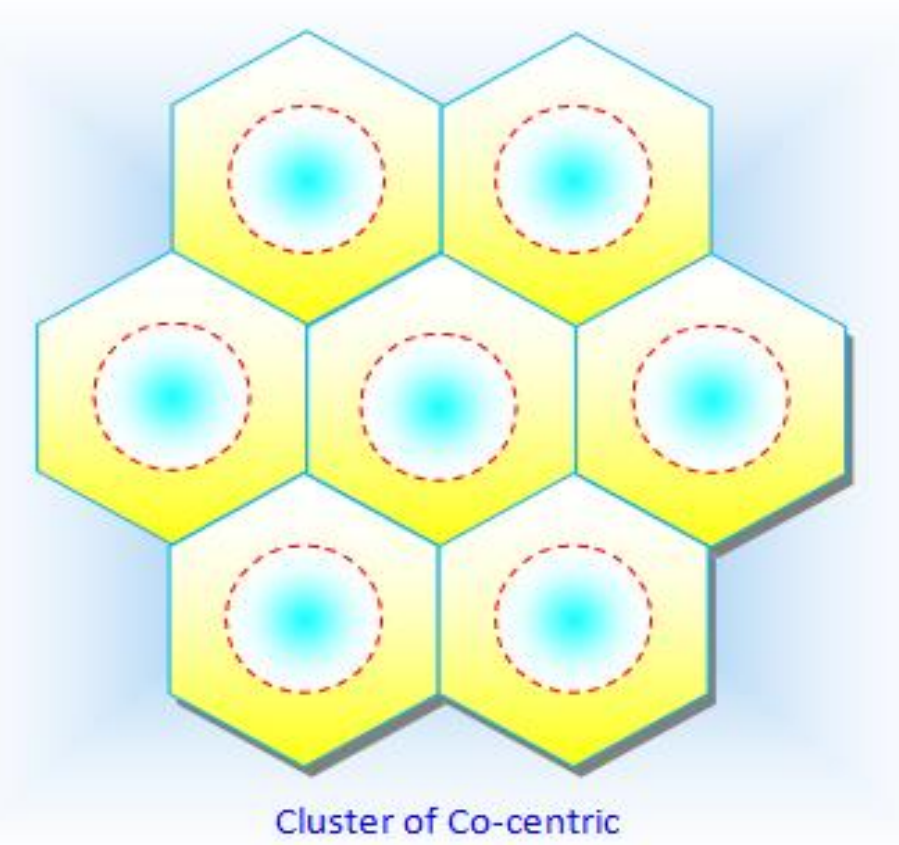

Figure 5. Cluster of Cells

For the new cells that are to be smaller in size, the transmit power of these cells must be reduced. The transmit power of the new cells with radius half that of the original cells can be found by examining the received power, Pr, at the Child and Mother cell boundaries and setting them equal to each other. This is necessary to ensure that the frequency reuse plan for the new microcell behaves exactly as for the original cells. From Figure 6, we have

$\operatorname{Pr}$.[Mother Cell] $\infty \mathrm{Pt}_{1} \mathrm{R}^{-\mathrm{n}}$ (1) and

Pr.[Child Cell] $\infty \mathrm{Pt}_{2}(\mathrm{R} / 2)^{-\mathrm{n}}$

Where $\mathrm{Pt}_{1}$ and $\mathrm{Pt}_{2}$ are the transmit powers of the larger and smaller cell base stations, respectively, and $\mathrm{n}$ is the path loss exponent. 
If we take $\mathrm{n}=4$ and set the received powers equal to each other,

Then $\mathrm{Pt}_{2}=\mathrm{Pt}_{1} / 16$

In other words, the transmit power must be reduced by $12 \mathrm{~dB}$ in order to fill in the original coverage area with microcell, while maintaining the $\mathrm{S} / \mathrm{I}$ requirement.

\subsection{Algorithm:}

Step 1: Location manager of inner cell is activated.

Step 2: $\quad$ IF it is not present in inner cell

THEN GOTO Step 4.

Step 3: After the call setup start scanning.

IF it find free channel allocation channel corresponding cell Handoff complete.

ELSE

Stop scanning go to Step 1 to Location manager and find the free channel Using location Manager Based on call duration, Signal Strength, Coverage area etc.

Find free channel and allocated the channel.

Step 4: Location manager of outer cell is activated.

Step 5: After the call setup start scanning.

IF it find free channel allocation channel corresponding cell Handoff complete.

\section{ELSE}

Stop scanning go to Location manager Step 4 and find the free channel Using location Manager Based on call duration, Signal Strength, Coverage area etc.

Find free channel and allocated the channel.

Step 6: Stop.

\section{Flow Chart:}

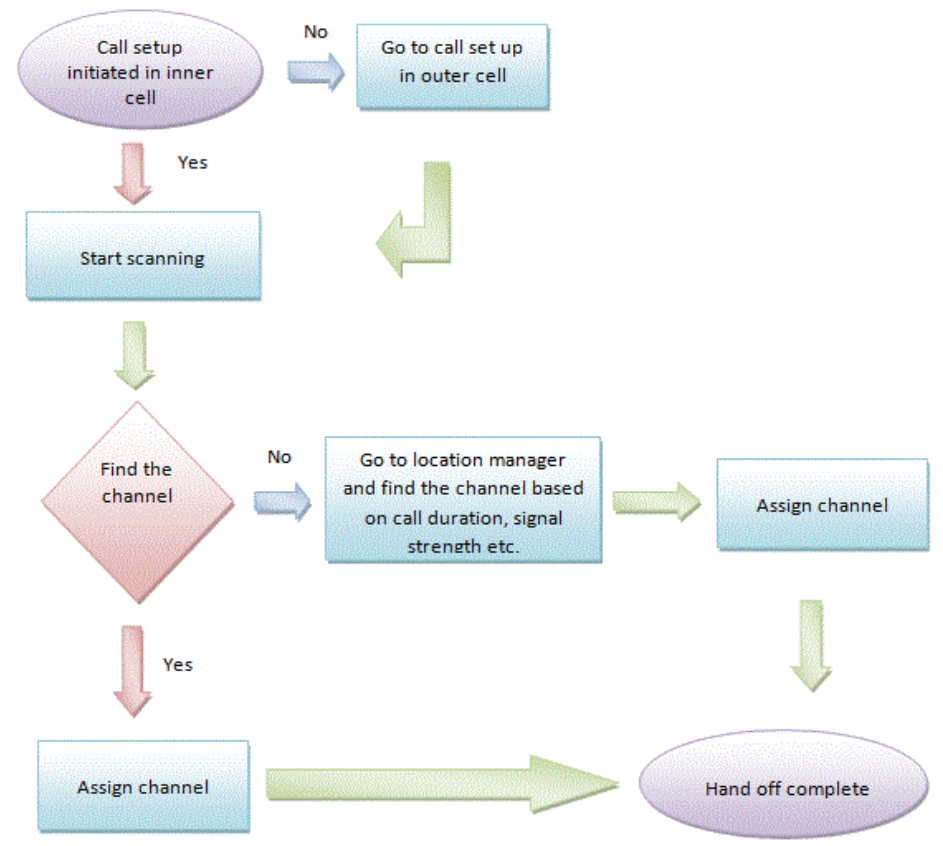

Figure 8 


\section{Simulation Result:}

Table 1

\begin{tabular}{|c|c|c|}
\hline S/no & Parameter & value \\
\hline $\mathbf{1}$ & Cluster(k) & 7 \\
\hline $\mathbf{2}$ & Total traffic Load & 500 \\
\hline $\mathbf{3}$ & Signal to interference ratio(s/i) & $18.99 \mathrm{db}$ \\
\hline
\end{tabular}

The probability that a new call is blocked given by the Erlang B formula was evaluated for different value of channels in ascending order using Mat lab. The corresponding values of call blocking probabilities are tabulated against the number of channels as shown below in Table 2 .

\section{Table 2}

\begin{tabular}{|c|c|c|}
\hline Sl no & Blocking probability & $\begin{array}{c}\text { No of } \\
\text { channel }\end{array}$ \\
\hline $\mathbf{1}$ & 0.9601 & 20 \\
\hline $\mathbf{2}$ & 0.9002 & 30 \\
\hline $\mathbf{4}$ & 0.8603 & 70 \\
\hline
\end{tabular}

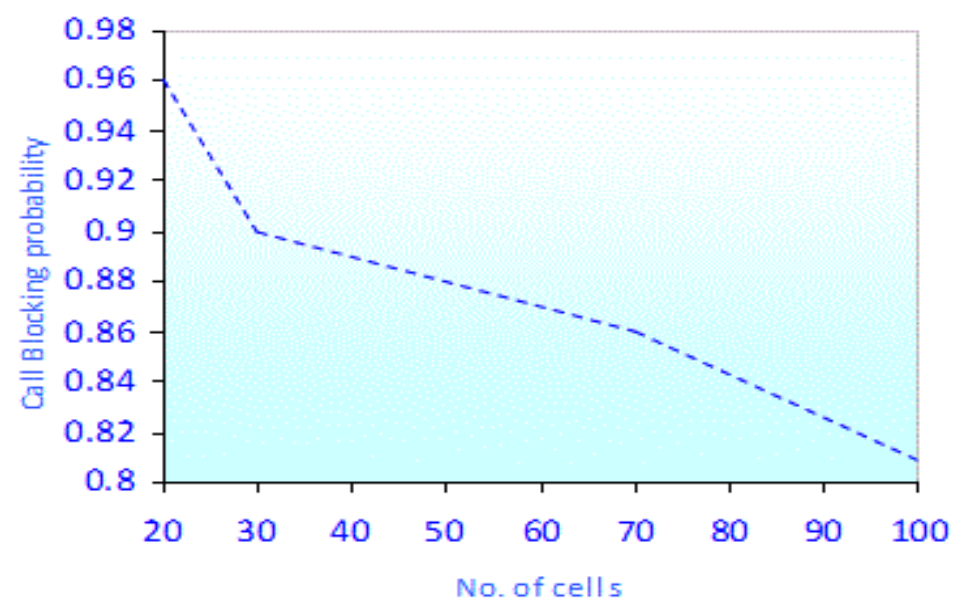

Figure 9. Graph of Blocking Probability against No of Channel

The probability that a new call is blocked represented as $\operatorname{Pr}[$ blocked] is plotted against channel capacity in Figure 9. The result shows that as the channel capacity increases, the call blocking probability $\operatorname{Pr}[$ blocked] reduces until a point when it becomes constant. Figure 9 proves that more calls are allowed in the system as the channel capacity increases. 


\section{Conclusion}

In conclusion, the capacity of a cellular communication system can be increased by using cell splitting and frequency reuse. The increment in channel capacity after cell splitting helps to reduce the call blocking probability. The results show that when properly and orderly carried out, the cell splitting technique has the capability of increasing the capacity of a congested cellular system.

\section{Future Works}

In our future work, we would try to find out that how to increase the channel capacity using genetic algorithm based approach.

\section{References}

[1] Hsookim, "Selective Channel Scanning for Fast Handoff in Wireless LAN using Neighbor Graph", Japan, July 2004.International Technical Conference on Circuits/Systems Computers and Communication.

[2] Cshengli, "A Neighbor Caching Mechanism For Handoff In IEEE802.11Wireless Networks", Springer, DOI 10.1007/S11227-008-0175-3, (2008) March 20.

[3] M. Shin, A. Mitra and W. A. Arbaugh, "Improving the Latency of 802.11 Handoffs Using Neighbour Graphs", Mobisys 'Proceedings Of The 2nd International Conference on Mobile Systems, Applications and Services, New York, USA, ACM Press, (2004), pp. 70-83.

[4] C. S. Li, "A Neighbor Caching Mechanism for Handoff in IEEE 802.11 Wireless Networks", Springer, DOI 10.1007/S11227-008-0175-3, (2008) March 20.

[5] S. Bavarian, "Symbol By Symbol Soft-Input Soft-Output Multiuser Detection for Frequency Selective MIMO Channels", Simon Fraser University Fall, (2008).

[6] "Displacement Of A High Altitude Platform On Cellular Interference And Handover", Submitted To IEEE Transactions On Wireless Communications, (2003) May.

[7] D. Everitt, "Traffic Capacity Of Cellular Mobile Communications Systems", Comp. Networks ISDN Sys., vol. 20, (1990), pp. 447-454.

[8] V. H. MacDonald, "The Cellular Concept", The Bell Technical Journal, vol. 58, no. 1, (2008) January.

\section{Authors}

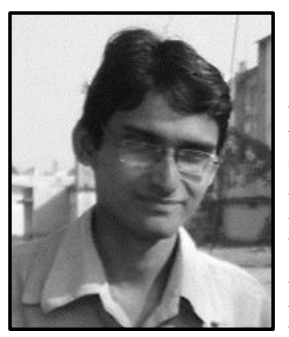

Ranjan Kumar Mondal received his M.Tech in Computer Science and Engineering from University of Kalyani, Kalyani, Nadia; and B.Tech in Computer Science and Engineering from Government College of Engineering and Textile Technology, Berhampore, Murshidabad, West Bengal under West Bengal University of Technology, West Bengal, India. At present, he is a Ph.D research scholar in Computer Science and Engineering from University of Kalyani. His research interests include Cloud Computing, Wireless and Mobile Communication Systems.

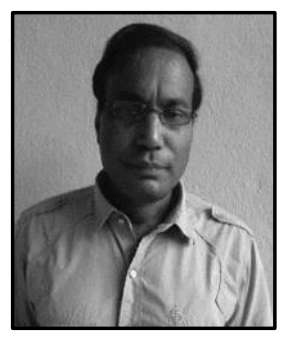

Debabrata Sarddar is an Assistant Professor at the Department of Computer Science and Engineering, University of Kalyani, Kalyani, Nadia, West Bengal, India. He completed his PhD from Jadavpur University. He did his M. Tech in Computer Science \& Engineering from DAVV, Indore in 2006, and his B.E in Computer Science \& Engineering from NIT, Durgapur in 2001. He has published more than 75 research papers in different journals and conferences. His research interests include Cloud Computing, Wireless and Mobile Communication Systems. 
International Journal of Future Generation Communication and Networking Vol. 8, No. 5 (2015) 